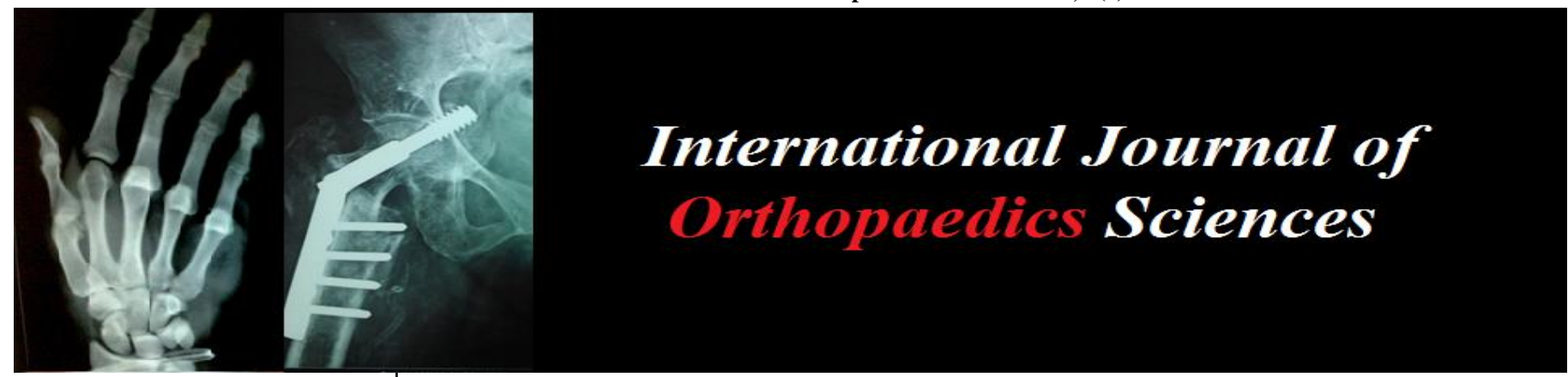

E-ISSN: 2395-1958

P-ISSN: 2706-6630

IJOS 2020; 6(4): 36-39

(C) $2020 \mathrm{IJOS}$

www.orthopaper.com

Received: 22-08-2020

Accepted: 25-09-2020

Ramesh Chandra Vadapalli Associate Professor, Department of Orthopaedics, Santhiram Medical College and General Hospital, Nandyal, Andhra Pradesh, India
Corresponding Author: Ramesh Chandra Vadapalli Associate Professor, Department of Orthopaedics, Santhiram Medical College and General Hospital, Nandyal, Andhra Pradesh, India

\section{Post-partum acute inflammatory sacroiliitis respond well to oral steroid and empirical antibiotic therapy}

\section{Ramesh Chandra Vadapalli}

DOI: https://doi.org/10.22271/ortho.2020.v6.i4a.2317

\section{Abstract}

Hip and low back pain is common during pregnancy or postpartum period. Herein, we report 12 cases with low back pain radiating to hip and buttocks presented to us after delivery and after failed NSAID therapy. MRI was very valuable and helped us in early diagnosis and promt treatment. We treated all patients with course of oral steroids and emperical antibiotic therapy in whom there was leuckocytosis. All patients responded very well to our treatment protocol. We recommend early MRI and oral steroids for fast recovery of postpartum - associated inflammatory sacroiliitis.

Keywords: Postpartum sacroilitis, backpain in pregnancy, postpartum hip pain

\section{Introduction}

Hip and low back pain is common during pregnancy or postpartum period. Pain may originate from the hip, lumbar or the sacroiliac joint (SIJ). Most of the Pain in the low back pain during or after pregnancy is usually nonspecific and a self-limiting. Differential diagnosis of low back pain and hip pain during pregnancy are transient hip osteoporosis, hip avascular necrosis, sacral stress fracture and postpartum sacroiliitis. post partum sacroilitis can be pyogenic and inflammatory.

\section{Materials and Methods}

Total of 46 cases of acute back pain and radiation to hip and lower limb were referred from department of obstetrics to our orthopaedic department from June 2017 to August 2019. Out of which 12 cases were referred tous after 10 days-2 weeks of conservative treatment with NSAIDS and physical therapy and rest. Out of 128 were normal delivery and 4 were caeserean sections. All caeserean sections were emergency. After a trial of labour. And all were prim is except one.

All patients presented with unilateral buttock pain and limp and unable to bear weight on the affected side. 7 were left and 5 were right. Pain was insidious in onset in 5 cases and acute in 7 cases. Only one patient had history of UTI prior to presentation. She was febrile for one day. Rest others were a febrile. None were diabetics or thyroid or prior history of sponylarthropathy. Average BMI was 25-28.

\section{Results}

On clinical examination there was localized tenderness on the affected sacroiliac joint. straight leg rising test was negative. The flexion, abduction, external rotation (FABERE) and Gaenslen tests were positive on the affected side. No swelling or local rise of temparature noticed. Average Erythrocyte sedimentation rate (ESR) was30- $40 \mathrm{~mm} /$ hour never exceeded $40 \mathrm{~mm}$. Creactive protein (CRP) was elevated in all cases ranging from 10 to $17 \mathrm{mg} / \mathrm{dL}$. In all cases there was leucocytosis in all cases but not more than 14000 . With neutrophil differential count more than 70 but less than 75. Urine normal examination was not significant. Initial radiographs of the pelvic and lumbar region were normal. We did Magnetic resonance imaging (MRI) of the lumbar spine and pelvis. We notice sacroiliac inflammatory changes with bone edema of iliac bone in 8 cases an in 4 cases we notice edema of ilacus muscles. 
Bone edema was typical around the upper SIJ, which was characterized by high intensity signal on the Short T1 Inversion Recovery (STIR) sequences and low-intensity signal on the T1-weighted sequences. Sclerosis was in the left iliac bone characterized by zones with low- intensity signal on T1- and T1-weighted sequences. Both sacroiliac articular surfaces were normal (Figures 1a, b) the patients were diagnosed with pregnancy-associated inflammatory sacroiliitis. We treated all patients with oral methyl prednisolone $4 \mathrm{mg}$ twice daily and empirical oral cephalosporin and paracetamol tid for one week. We also recommended cold pack application to the sacroiliac region twice a day. The patients were instructed to perform bed exercises (ankle rotation-pumps, knee bends, quadriceps sets, hip abduction-extension sets). All patients responded very well to course of steroids an antibiotics. Recovery time was between 7 to 20 days. But noticeable and satisfactory recovery was seen at the end of one week. At three months follow-up, patients were able to perform all daily activities without pain.
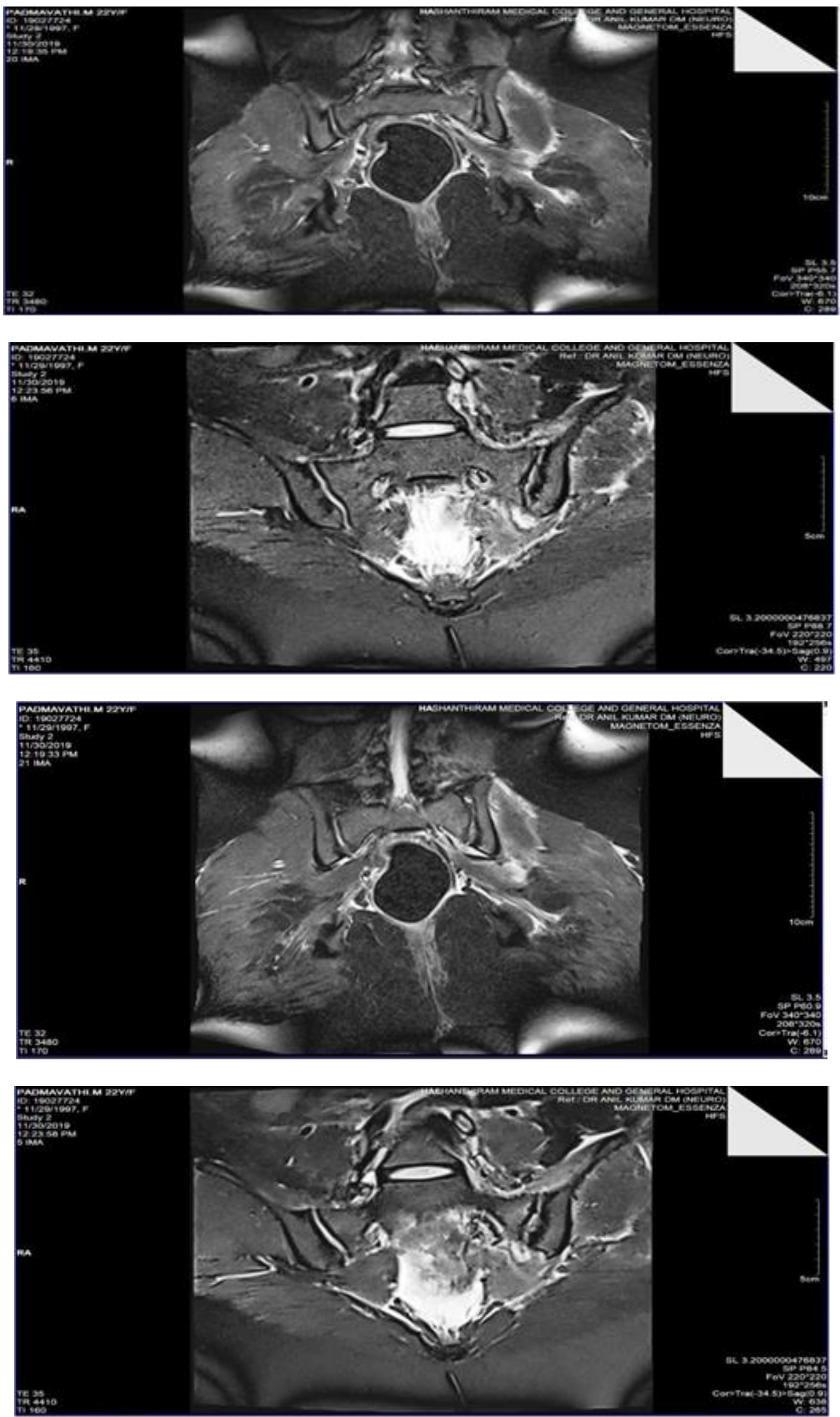


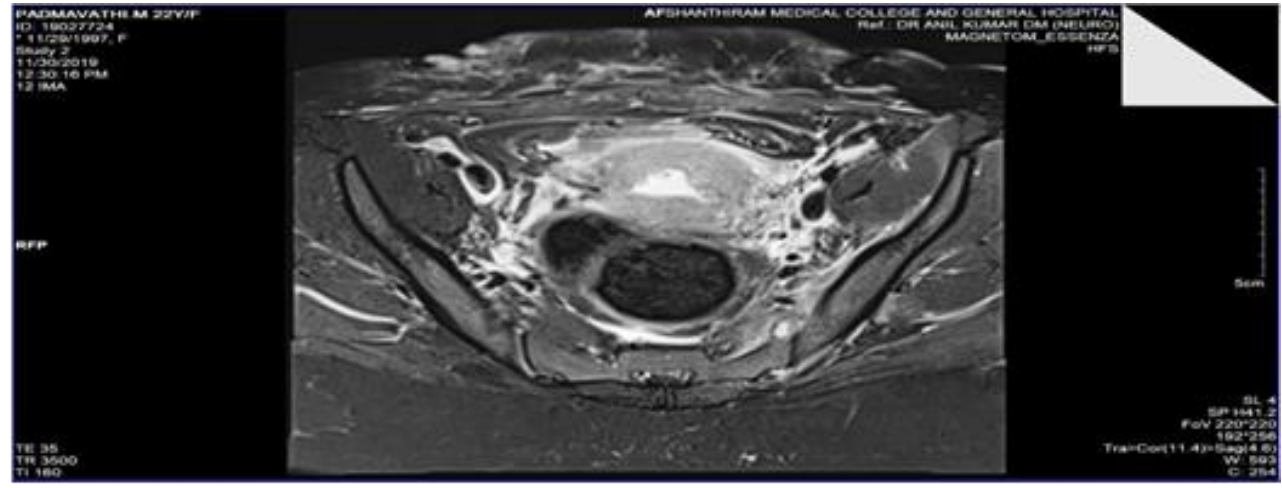

Fig 1: (a) Bone edema (high-intense) in a triangular shape and sclerosis (low-intense) in the left iliac bone on short T1 inversion recovery sequences. (b) Bone edema and sclerosis characterized by low-intensity on T1-weighted sequences

\section{Discussion}

Postpartum inflammatory sacroilitis is a not so uncommon. Till date only few case reports were published in literature.

Weight gain, hormonal, and postural changes associated with pregnancy may disrupt the pelvic stability ${ }^{[4]}$. Increased lumbar lordosis, pelvic tilt to anterior, and softening of the ligaments by relaxin give rise to biomechanical changes in the pelvic region ${ }^{[4]}$. Sacroiliac joint is a non-weight-bearing joint with a small range of motion which has to endure extraburden during pregnancy due to biomechanical changes, thereby, leading to pubic instability, inflammation, bone edema, and stress fractures ${ }^{[5]}$. The latter can easily occur, if there is also accompanying insufficient bone mass due to osteopenia or vitamin D deficiency ${ }^{[5]}$. The first case had severe pain and her CRP was very high, leading us to include pyogenic sacroiliitis was reported stress fracture was reported. In MRI, bone edema with no sign of erosion or destruction in the SIJ (Figures 2a, b) MRI showed a vertical fissure surrounded by medullary bone edema in the right sacrum involving the sacral ala and medial to the SIJ, which was diagnosed with sacral stress fracture.

Conservative treatment with oral and parenteral NSAIDS, Physical therapy with intermittent ultrasound, magneto therapy, hot cold packs, and electrostimulation were initial mode of treatments. But the recovery was slow in some series. Vitamin D replacement therapy was given, as her serum 25hydroxy vitamin D [25(OH) D] level was very low (7.4 $\mathrm{ng} / \mathrm{mL}$ ). All patients were instructed to wear a lumbosacral corset during ambulation.

Krishna Kumar et al. reported case of Tuberculous sacroilitis of post partum. Pyogenic sacroiliitis may be accompanied by a history of intravenous drug abuse, infective endocarditis, urinary tract infection, abortion or delivery ${ }^{[6]}$. There is commonly high fever along with severe back pain. Laboratory findings include leukocytosis, elevated ESR and CRP levels, and occasionally positive blood and urine cultures ${ }^{[6]}$. Bone edema, sclerosis, and erosions in SIJ are typical radiological changes similar to those in postpartum sacroiliitis ${ }^{[7]}$. we in our patients have not seen erosions of the Si joint. But bone marrow enema and edema of surrounding musculature was significant fining seven cases had significant leuckocytosis but not more than 1400. Elevated CRP could possibly be due to the recent caesarean section ${ }^{[8]}$. Most importantly, the sacroiliac articular surfaces were normal on the MRI. Spondyloarthritis was included in the differential diagnosis of our cases. The personal and family histories were negative for chronic back pain, stiffness, peripheral arthritis, inflammatory bowel disease, or uveitis. Although spondyloarthritis may flare up during the postpartum period, the symptoms started during the past days of pregnancy in our case, which is atypical of a flare-up. Additionally, the upper part of the SIJ was affected in most of our cases, as opposed to the typical dorsocaudal synovial involvement in early sacroiliitis of spondyloarthritis ${ }^{[9]}$.

Although the patient's history and clinical presentation were atypical for spondy loarthritis (SPA), axial SPA was unable to be excluded, since our case had sacroiliitis. Therefore, SPA should be considered during follow-up. Osteitiscondensansilii (OCI), a condition characterized by bilateral well defined triangular sclerosis of SIJ, was also considered in the differential diagnosis. This clinically benign condition predominantly affects the iliac portion of the SIJ, and the acute phase reactants are usually within normal limits ${ }^{[10]}$. However we excluded this diagnosis, as because laboratory parameters were not in normal limits and radiological involvement of the SIJ were not typical for OCI. Sacral stress fractures usually occur in elderly with osteoporosis or in individuals with a normal bone density who are exposed to repetitive stress, such as in long-distance runners or marching soldiers ${ }^{[2,11]}$.

Biomechanical and hormonal (Relaxin induced) changes during pregnancy may also cause bone edema and sacral stress fractures, in particular, in the presence of insufficient bone mass due to osteopenia or vitamin D deficiency ${ }^{[12]}$. Sacral stress fractures most commonly involve the sacral ala and are located medial to the SIJs and may be uni or bilateral [13]. In our case, the location of the lesion was typical for a sacral stress fracture. In the literature, both uni- and bilateral fractures are reported with relatively equal frequencies ${ }^{[14]}$.

\section{Conclusion}

Hip and low back pain is common during pregnancy or postpartum period but postpartum sacroilitis is were uncommon which knees careful analysis of the clinical, laboratory, and radiographic findings may help the clinician to detect the underlying cause and tailor an appropriate treatment. When initial treatment of NSAIDS fail we recommend steroids and oral antibiotics to recover fast and resume daily activities.as most of the women are breast feeding we strongly recommend steroids for fast recovery. We recommend early MRI and prompt referral to orthopaeician if any hip and low back pain doesn't subside in $72 \mathrm{hrs}$ of initial conservative treatment.

Declaration of conflicting interests. The authors declared no conflicts of interest with respect to the authorship and/or publication of this article.

Funding: The authors received no financial support for the research and/or authorship of this article. 


\section{References}

1. Ostgaard HC, Andersson GB, Karlsson K. Prevalence of back pain in pregnancy. Spine (Phila Pa 1976). 1991; 16:549-52.

2. Norén L, Ostgaard S, Johansson G, Ostgaard HC. Lumbar back and posterior pelvic pain during pregnancy: a 3-year follow-up. Eur Spine J. 2002; 11:267-71.

3. Spigset $\mathrm{O}$, Hägg S. Analgesics and breast-feeding: safety considerations. Paediatr Drugs. 2000; 2:223-38.

4. Arikan E, Ozcan E. Painful conditions arising from musculoskeletal system during pregnancy and therapeutic management. Turk J Phys Med Rehab. 2005; 51:65-8.

5. Thienpont E, Simon JP, Fabry G. Sacral stress fracture during pregnancy--a case report. Acta Orthop Scand. 1999; 70:525-6.

6. Almoujahed MO, Khatib R, Baran J. Pregnancyassociated pyogenic sacroiliitis: case report and review. Infect Dis Obstet Gynecol. 2003; 11:53-7.

7. Karunakaran M. Postpartum sacroiliitis. Available from: http://thirdeye- radiologysite.blogspot.com.tr/2012/06/ postpartum-sacroiliitis.html.

8. Keski-Nisula L, Kirkinen P, Ollikainen M, Saarikoski S. C-reactive protein in uncomplicated parturients delivered by cesarean section. Acta Obstet Gynecol Scand. 1997; 76:862-7.

9. Muche B, Bollow M, François RJ, Sieper J, Hamm B, Braun J. Anatomic structures involved in early- and latestagesacroiliitis in spondylarthritis: a detailed analysis by contrast- enhanced magnetic resonance imaging. Arthritis Rheum. 2003; 48:1374-84.

10. Mitra R. Osteitis CondensansIlii. RheumatolInt. 2010; 30:293-6.

11. Thienpont E, Simon JP, Fabry G. Sacral stress fracture during pregnancy--a case report. Acta Orthop Scand. 1999; 70:525-6.

12. Longhino V, Bonora C, Sansone V. The management of sacral stress fractures: current concepts. Clin Cases Miner Bone Metab. 2011; 8:19-23.

13. Lyders EM, Whitlow CT, Baker MD, Morris PP. Imaging and treatment of sacral insufficiency fractures. AJNR Am J Neuroradiol. 2010; 31:201-10.

14. De Smet AA, Neff JR. Pubic and sacral insufficiency fractures: clinical course and radiologic findings. AJR Am J Roentgenol. 1985; 145:601-6. 